\title{
THE VALUED GABRIEL QUIVER OF A WEDGE PRODUCT AND SEMIPRIME COALGEBRAS
}

\author{
GABRIEL NAVARRO
}

\begin{abstract}
We make a first approach to the representation theory of the wedge product of coalgebras by means of the description of its valued Gabriel quiver. Then we define semiprime coalgebras and study its category of comodules by the use of localization techniques. In particular, we prove that, whether its Gabriel quiver is locally finite, any monomial semiprime fc-tame coalgebra is string. We also prove a weaker version of Eisenbud-Griffith theorem for coalgebras, namely, any hereditary semiprime strictly quasi-finite coalgebra is serial.
\end{abstract}

\section{INTRODUCTION}

Throughout this paper we fix a field $k$ and denote by $C$ a $k$-coalgebra. Unless otherwise stated, we shall assume that $C$ is basic [5] [29], that is, $C$ and its (left or right) socle have decompositions

$$
C=\bigoplus_{i \in I_{C}} E_{i} \quad \text { and } \quad \operatorname{soc} C=\bigoplus_{i \in I_{C}} S_{i}
$$

where $\left\{E_{i}\right\}_{i \in I_{C}}$ is a complete set of pairwise non-isomorphic indecomposable injective right $C$-comodules and $\left\{S_{i}\right\}_{i \in I_{C}}$ is a complete set of pairwise non-isomorphic simple right (and left) $C$-comodules.

Given two subcoalgebras $A$ and $B$ of $C$, the wedge product [38] of $A$ and $B$ in $C$, $A \wedge^{C} B=\operatorname{Ker}(C \stackrel{\Delta}{\longrightarrow} C \otimes C \stackrel{p r \otimes p r}{\longrightarrow} C / A \otimes C / B)$, where $\Delta$ is the comultiplication of $C$ and $p r$ is the standard projection. Equivalently, $A \wedge^{C} B$ is the set of elements $x \in C$ such that $\Delta(x) \in A \otimes C+C \otimes B$, or equivalently, $A \wedge^{C} B=\left(A^{\perp} B^{\perp}\right)^{\perp}$, where $\perp$ denotes the standard orthogonality. If there is no ambiguity, we simply denote the wedge product by $A \wedge B$.

In the "coalgebraic" setting, prime coalgebras, as those which cannot been decomposed non-trivially as a wedge product, has been investigated by several authors. The notion appeared first in Takeuchi's PhD Thesis [39] under cocommutative conditions. Nevertheless, as it was pointed out in [18], it could be defined in the same way without this restriction. This is done in [25], where the authors analyze the Zariski topology attached to the set of prime subcoalgebras over a field. In [18], pointed prime coalgebras over a field are studied from the graphical point of view of path coalgebras of a quiver. In a more general setting, it has been developed in the context of coalgebras over a commutative ring [41] under the name of wedge coprime coalgebras; corings [2] [3], calling them fully coprime corings; or

Research supported by Spanish MEC project MTM2007-66666, and TIC-111 (Junta de Andalucía Research Group). 
modules and comodules 2] [9]; suffering progressive generalizations which, whether the base ring is a field, coincide with the notion given by Takeuchi. Nevertheless, unless in a very tangential way, none of the above papers deals with the category of comodules of a prime coalgebra or coring.

In this paper we make a first approach to the representation theory of a wedge product of arbitrary coalgebras over a field and describe its right valued Gabriel quiver [19] (Theorem 2.4). From this point of view, we deal with semiprime coalgebras proving that its valued Gabriel quiver should have an specific shape (Theorem 3.6), as a generalization of a result obtained in [18]. As a consequence, we give a weaker version of the coalgebraic analog of a theorem of Eisenbud and Griffith [8] proven in [14] (Corollary 3.7). In the last section, we shall apply these results in order to get certain properties of the category of comodules of a semiprime coalgebra. In particular, we highlight that, over an algebraically closed field, a monomial semiprime fc-tame coalgebra whose Gabriel quiver is locally finite is string in the sense of [30] (Theorem 4.8). We would like to remark that, from this perspective, semiprime coalgebras seems to be a more appropriated class than prime coalgebras since this class is closed under direct sums.

We also would like to remark that we follow the nomenclature of [18] and call the coalgebras "prime" or "semiprime", whilst the papers above-mentioned make use of the word "coprime" or "cosemiprime". We think that add the prefix "co" in "coalgebra" is enough for pointing out the dual nature of this notion and makes more readable the manuscript. The reader also should note that prime coalgebras over a field as described in [9] differ from the ones treated here, since all of them are simple.

All along the paper we shall make use of the localization techniques develop in [16], [17], 24], 31] or [33] which have been showed to be an efficient tool for developing the Representation Theory of Coalgebras. Actually, we partially solve a subtle mistake in the proof of [18, Theorem 4.2]. Therefore, for the convenience of the reader, let us remind the localization theory in category of comodules. Throughout we denote by $\mathcal{M}_{f}^{C}, \mathcal{M}_{q f}^{C}$ and $\mathcal{M}^{C}$ the category of finite dimensional, quasi-finite and all right $C$-comodules, respectively.

Let $\mathcal{T}$ be a dense subcategory (or a Serre class) of the category $\mathcal{M}^{C}, \mathcal{T}$ is said to be localizing (cf. [10]) if the quotient functor $T: \mathcal{M}^{C} \rightarrow \mathcal{M}^{C} / \mathcal{T}$ has a right adjoint functor $S$, called the section functor. If the section functor is exact, $\mathcal{T}$ is called perfect localizing.

From the general theory of localization in Grothendieck categories [10], it is well-known that there exists a one-to-one correspondence between localizing subcategories of $\mathcal{M}^{C}$ and sets of indecomposable injective right $C$-comodules, and, as a consequence, sets of simple right $C$-comodules. More precisely, a localizing subcategory is determined by an injective right $C$-comodule $E=\oplus_{j \in J} E_{j}$, where $J \subseteq I_{C}$ (therefore the associated set of indecomposable injective comodules is $\left.\left\{E_{j}\right\}_{j \in J}\right)$. Then $\mathcal{M}^{C} / \mathcal{T} \simeq \mathcal{M}^{D}$, where $D$ is the coalgebra of coendomorphism $\operatorname{Cohom}_{C}(E, E)$ (cf. [40] for definitions), and the quotient and section functors are $\operatorname{Cohom}_{C}(E,-)$ and $-\square_{D} E$, respectively.

In [7], [18] and [42], localizing subcategories are described by means of idempotents in the dual algebra $C^{*}$. In particular, it is proved that the quotient category $\mathcal{M}^{C} / \mathcal{T}$ is the category of right comodules over the coalgebra $e C e$, where $e \in C^{*}$ is an idempotent associated to the localizing subcategory $\mathcal{T}$ (that is, $E=C e$, where $E$ is the injective right $C$-comodule associated to the localizing subcategory $\mathcal{T}$ ). The coalgebra structure of $e C e$ 
(cf. [26]) is given by

$$
\Delta_{e C e}(e x e)=\sum_{(x)} e x_{(1)} e \otimes e x_{(2)} e \quad \text { and } \quad \epsilon_{e C e}(e x e)=\epsilon_{C}(x)
$$

for any $x \in C$, where $\Delta_{C}(x)=\sum_{(x)} x_{(1)} \otimes x_{(2)}$ using the sigma-notation of [38]. For completeness, we recall from [7] (see also [18]) the following description of the localizing functors. We recall that, given an idempotent $e \in C^{*}$, for each right $C$-comodule $M$, the vector space $e M$ is endowed with a structure of right $e C e$-comodule given by

$$
\rho_{e M}(e x)=\sum_{(x)} e x_{(1)} \otimes e x_{(0)} e
$$

where $\rho_{M}(x)=\sum_{(x)} x_{(1)} \otimes x_{(0)}$ using the sigma-notation of [38].

The localization in categories of comodules over path coalgebras is described in detail in [16] and [18]. Briefly, following the notation of these papers, let $Q=\left(Q_{0}, Q_{1}\right)$ be a (possibly infinite) quiver. The path algebra $k Q$ can be endowed with a coalgebra structure with comultiplication given by

$$
\Delta(p)=e_{j} \otimes p+p \otimes e_{i}+\sum_{i=1}^{m-1} \alpha_{m} \cdots \alpha_{i+1} \otimes \alpha_{i} \cdots \alpha_{1}=\sum_{\eta \tau=p} \eta \otimes \tau
$$

for any path $p=\alpha_{m} \cdots \alpha_{1}$ in $Q$ from $e_{i}$ to $e_{j}$, and for a trivial path, $e_{i}, \Delta\left(e_{i}\right)=e_{i} \otimes e_{i}$.

Given $X \subseteq Q_{0}$, a path $p=\alpha_{n} \cdots \alpha_{1}$ in $Q$ is said to be a cell in $Q$ relative to $X$ (shortly a cell) if $s\left(\alpha_{1}\right), t\left(\alpha_{m}\right) \in X$ and $s\left(\alpha_{i}\right) \notin X$ for all $i=2, \ldots, n-1$, where $s(\alpha)$ and $t(\alpha)$ denote the source and the sink of an arrow or a path $\alpha$.

The localizing subcategories of $\mathcal{M}^{k Q}$ are in one-to-one correspondence with subsets of vertices of $Q$ and also, in one-to-one correspondence with idempotents of the dual algebra $(k Q)^{*}$. Then, given $X_{e} \subseteq Q_{0}$ corresponding to an idempotent $e \in(k Q)^{*}, e(k Q) e \cong k Q^{e}$, where $\left(Q^{e}\right)_{0}=X_{e}$ and the arrows in $Q^{e}$ from a vertex $x$ to a vertex $y$ is the number of different cells relative to $X_{e}$ between these vertices, see [16].

\section{The valued Gabriel Quiver of a Wedge Product}

The valued Gabriel quiver of a coalgebra carries the information of the second piece of its coradical filtration. Therefore, it used to be one of the first invariant under consideration when dealing with its representation theory. Following [19], let us recall that the right valued Gabriel quiver $\left(Q_{C}, d_{C}\right)$ of a basic coalgebra $C$ as follows: the set of vertices of $\left(Q_{C}, d_{C}\right)$ is the set of simple right $C$-comodules $\left\{S_{i}\right\}_{i \in I_{C}}$, and there exists a unique valued arrow

$$
S_{i} \stackrel{\left(d_{i j}^{\prime}, d_{i j}^{\prime \prime}\right)}{\longrightarrow} S_{j}
$$

if and only if $\operatorname{Ext}_{C}^{1}\left(S_{i}, S_{j}\right) \neq 0$ and,

$$
d_{i j}^{\prime}=\operatorname{dim}_{G_{i}} \operatorname{Ext}_{C}^{1}\left(S_{i}, S_{j}\right) \text { and } d_{i j}^{\prime \prime}=\operatorname{dim}_{G_{j}} \operatorname{Ext}_{C}^{1}\left(S_{i}, S_{j}\right),
$$

as a right $G_{i}$-module and as a left $G_{j}$-module, respectively. When the values of the arrows are irrelevant for our purposes, we shall denote the valued Gabriel quiver of $C$ simply by $Q_{C}$. 
In [31, the valued Gabriel quiver of $C$ is described through the notion of irreducible morphisms between indecomposable injective right $C$-comodules. Let us denote by inj ${ }^{C}$ (respect. ${ }^{C}$ inj) the full subcategory of $\mathcal{M}^{C}$ (respect. ${ }^{C} \mathcal{M}$ ) formed by socle-finite (i.e., comodules whose socle is finite-dimensional) injective right (respect. left) $C$-comodules. Let $E$ and $E^{\prime}$ be two comodules in inj ${ }^{C}$. A morphism $f: E \rightarrow E^{\prime}$ is said to be irreducible if $f$ is not an isomorphism and given a factorization

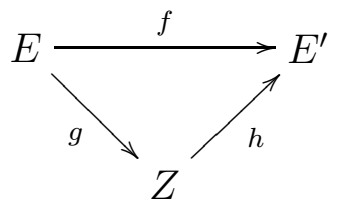

of $f$, where $Z$ is in inj ${ }^{C}, g$ is a section, or $h$ is a retraction. Analogously to the case of finitedimensional algebras, there it is proven that the set of irreducible morphism $\operatorname{Irr}_{C}\left(E_{i}, E_{j}\right)$ between two indecomposable injective right $C$-comodules $E_{i}$ and $E_{j}$ is isomorphic, as $G_{j}-G_{i}$-bimodule, to the quotient $\operatorname{rad}_{C}\left(E_{i}, E_{j}\right) / \operatorname{rad}_{C}^{2}\left(E_{i}, E_{j}\right)$. We recall that, for each two indecomposable injective right $C$-comodules $E_{i}$ and $E_{j}$, the radical of $\operatorname{Hom}_{C}\left(E_{i}, E_{j}\right)$ is the $K$-subspace $\operatorname{rad}_{C}\left(E_{i}, E_{j}\right)$ of $\operatorname{Hom}_{C}\left(E_{i}, E_{j}\right)$ generated by all non-isomorphisms. Observe that if $i \neq j$, then $\operatorname{rad}_{C}\left(E_{i}, E_{j}\right)=\operatorname{Hom}_{C}\left(E_{i}, E_{j}\right)$. The square of $\operatorname{rad}_{C}\left(E_{i}, E_{j}\right)$ is defined to be the $K$-subspace

$$
\operatorname{rad}_{C}^{2}\left(E_{i}, E_{j}\right) \subseteq \operatorname{rad}_{C}\left(E_{i}, E_{j}\right) \subseteq \operatorname{Hom}_{C}\left(E_{i}, E_{j}\right)
$$

generated by all composite homomorphisms of the form

$$
E_{i} \stackrel{f}{\longrightarrow} E_{k} \stackrel{g}{\longrightarrow} E_{j},
$$

where $f \in \operatorname{rad}_{C}\left(E_{i}, E_{k}\right)$ and $g \in \operatorname{rad}_{C}\left(E_{k}, E_{j}\right)$. The $m$ th power $\operatorname{rad}_{C}^{m}\left(E_{i}, E_{j}\right)$ of $\operatorname{rad}_{C}\left(E_{i}, E_{j}\right)$ is defined analogously, for each $m>2$.

Let us now prove a generalization of [23, Theorem 1.7] which describes the right valued Gabriel quiver by means of the wedge product of simple right comodules.

Proposition 2.1. Let $C$ be a coalgebra and, $S_{i}$ and $S_{j}$ be two simple right $C$-comodules. There exists an arrow in $\left(Q_{C}, d_{C}\right)$ from $S_{j}$ to $S_{i}$ if and only if $\left(S_{i} \wedge \wedge^{C} S_{j}\right) /\left(S_{i}+S_{j}\right) \neq 0$. In such a case, the arrow is labeled by $d_{j i}=\left(d_{j i}^{1}, d_{j i}^{2}\right)$, where $d_{j i}^{1}=\operatorname{dim}_{G_{j}}\left(S_{i} \wedge^{C} S_{j}\right) /\left(S_{i}+S_{j}\right)$ as right $G_{j}$-comodule, and $d_{j i}^{2}=\operatorname{dim}_{G_{i}}\left(S_{i} \wedge^{C} S_{j}\right) /\left(S_{i}+S_{j}\right)$ as left $G_{i}$-comodule.

Proof. Following [6, Lemma 3.9], for any right $C$-comodule $I$ and any subcoalgebra $E$, there exists an isomorphism $\left(I \wedge^{C} E\right) / I \cong C / I \square_{C} E$ of right $E$-comodules. Therefore,

$$
\frac{S_{i} \wedge^{C} S_{j}}{S_{i}} \cong C / S_{i} \square_{C} S_{j} \cong\left(\oplus_{k \neq i} E_{k} \square_{C} S_{j}\right) \oplus E_{i} / S_{i} \square_{C} S_{j}
$$

In general, for any right $C$-comodule $M, M \square_{C} S_{j}$ is the direct sum of the simple right $C$-comodules isomorphic to $S_{j}$ appearing in the socle of $M$. Therefore $\oplus_{k \neq i} E_{k} \square_{C} S_{j} \cong S_{j}$ if $j \neq i$ and zero otherwise. Furthermore, $E_{i} / S_{i} \square_{C} S_{j} \cong S_{j}^{(r)}$, where

$$
r=\operatorname{dim}_{G_{j}} \operatorname{Hom}_{C}\left(S_{j}, E_{i} / S_{i}\right)=\operatorname{dim}_{G_{j}} \operatorname{Ext}^{C}\left(S_{j}, S_{i}\right)
$$


see [24]. Now,

$$
\frac{S_{i} \wedge S_{j}}{S_{i}+S_{j}} \cong \frac{\left(S_{i} \wedge S_{j}\right) / S_{i}}{\left(S_{i}+S_{j}\right) / S_{i}} \cong S_{j}^{(r)}
$$

as right $S_{j}$-comodules. Then, the equivalence and the calculation of the first component of the label hold.

We recall from [14] or [20] that the right valued Gabriel quiver and the left valued Gabriel quiver of $C$ are opposite one to each other. Now, it is enough to apply the left version of the formula of [6, Lemma 3.9] and a similar reasoning as above in order to calculate $d$ and prove the statement.

This can be generalized taking into account the notion of predecessor defined in [24]. We remind that, given a simple $C$-comodule $S_{i}$, we say that a simple $C$-comodule $S_{j}$ is an $n$-predecessor of $S_{i}$ if $\operatorname{Ext}_{C}^{1}\left(S_{j}, \operatorname{soc}^{n} E_{i}\right) \neq 0$ for some $n>0$, or equivalently, if $S_{j} \subseteq \operatorname{soc}\left(E_{i} / \operatorname{soc}^{n} E_{i}\right) \cong \operatorname{soc}^{n+1} E_{i} / \operatorname{soc}^{n} E_{i}$ for some $n>0$, where $E_{i}$ is the injective envelope of $S_{i}$.

Proposition 2.2. Let $C$ be a coalgebra and, $S_{i}$ and $S_{j}$ be two simple right $C$-comodules. $S_{j}$ is a n-predecessor of $S_{i}$ if and only if $\left(\operatorname{soc}^{n} S_{i} \wedge{ }^{C} S_{j}\right) /\left(\operatorname{soc}^{n} S_{i}+S_{j}\right) \neq 0$. In such a case, the number of indecomposable direct summands of $\operatorname{soc}^{n+1} E_{i} / \mathrm{soc}^{n} E_{i}$ isomorphic to $S_{j}$ is $\operatorname{dim}_{G_{j}}\left(\operatorname{soc}^{n} S_{i} \wedge^{C} S_{j}\right) /\left(\operatorname{soc}^{2} S_{i}+S_{j}\right)$ as right $G_{j}$-comodule.

Proof. It is similar to the former proof. Simply consider that

$$
\frac{\operatorname{soc}{ }^{n} S_{i} \wedge{ }^{C} S_{j}}{\operatorname{soc}^{n} S_{i}} \cong C / \operatorname{soc}{ }^{n} S_{i} \square_{C} S_{j} \cong\left(\oplus_{k \neq i} E_{k} \square_{C} S_{j}\right) \oplus E_{i} / \operatorname{soc}{ }^{n} S_{i} \square_{C} S_{j}
$$

and $\operatorname{soc}\left(E_{i} / \operatorname{soc}^{n} S_{i}\right)=\operatorname{soc}^{n+1} E_{i} / \operatorname{soc}^{n} E_{i}$.

Proposition 2.3. Let $C$ be a coalgebra and, $A$ and $B$ two subcoalgebras of $C$. Then the following assertions hold:

(a) Let $\mathfrak{G}_{A}=\left\{S_{j}\right\}_{j \in I_{A}}, \mathfrak{G}_{B}=\left\{S_{k}\right\}_{k \in I_{B}}$ and $\mathfrak{G}_{A \wedge C} B=\left\{S_{i}\right\}_{i \in I_{A \wedge B}}$ be a complete set of pairwise non-isomorphic simple right $A$-comodules, $B$-comodules and $A \wedge^{C} B$-comodules, respectively. Then $\mathfrak{G}_{A \wedge}{ }^{C} B=\mathfrak{G}_{A} \cup \mathfrak{G}_{B}$.

(b) Given two simple $A \wedge^{C} B$-comodules $S$ and $T$ :

$$
S \wedge^{A \wedge B} T= \begin{cases}S \oplus T & \text { if } S \nsubseteq A \text { and } T \nsubseteq B \\ S \wedge^{C} T & \text { if } S \subseteq A \text { and } T \subseteq B \\ S \wedge^{A} T & \text { if } S \subseteq A \text { and } T \nsubseteq B \\ S \wedge^{B} T & \text { if } S \nsubseteq A \text { and } T \subseteq B\end{cases}
$$

Proof. (a) It is obvious that $\mathfrak{G}_{A} \cup \mathfrak{G}_{B} \subseteq \mathfrak{G}_{A \wedge^{C} B}$ since $A$ and $B$ are subcoalgebras of $A \wedge{ }^{C} B$. Let now $S$ be a simple right $A \wedge^{C} B$-comodule. Then $\Delta(S) \subseteq S \otimes\left(A \wedge^{C} B\right)$ and, since $S \subseteq A \wedge^{C} B, \Delta(S) \subseteq A \otimes C+C \otimes B$. Now, if $S \subseteq A, S$ is a $A$-comodule. If not, $S \cap A=\emptyset$ so $\Delta(S) \subseteq S \otimes B$, and hence $S$ is a $B$-comodule.

(b) If $S \subseteq A$ and $T \subseteq B, S \wedge^{C} T \subseteq A \wedge^{C} B$, and therefore

$$
S \wedge^{A \wedge B} T=\left(S \wedge^{C} T\right) \cap\left(A \wedge^{C} B\right)=S \wedge^{C} T \text {. }
$$

Assume that $S \nsubseteq A$ and $T \nsubseteq B$ and let $0 \rightarrow S \rightarrow M \rightarrow T \rightarrow 0$ be a non-split short exact sequence of $A \wedge^{C} B$-comodules. Then $M$ is a serial $A \wedge^{C} B$-comodule with simple 
socle $A \subseteq B$. In particular, $A \cap M=0$. Since $M \subset A \wedge^{C} B$ and $\Delta(M) \subseteq M \otimes C$, it follows that $\Delta(M) \subseteq M \otimes B$ and so $M$ is a right $B$-comodule. Hence $S$ is a right $B$-comodule and we get a contradiction. Thus $\operatorname{Ext}^{A \wedge B}(T, S)=0$. In particular $S \wedge^{A \wedge B} T=S \oplus T$.

Let us suppose that $S \subseteq A$ and $T \nsubseteq B$, and $x \in S \wedge^{A \wedge B} T$. Then we may write $\Delta(x)=\sum x_{i} \otimes y_{i}+\sum x_{j} \otimes y_{j}$, where $x_{i} \in S, y_{j} \in T$ and $y_{i}, x_{j} \in A \wedge B$. Nevertheless $x \in A \wedge^{C} B$ and so $\Delta(x) \in A \otimes C+C \otimes B$. Since $T \nsubseteq B$, we deduce that the elements $x_{j} \in A$. Thus $\Delta(x) \in A \otimes\left(A \wedge^{C} B\right)$ and $x=(i d \otimes \epsilon) \Delta(x) \in A$. Hence $x \in S \wedge^{A} T$. Analogously, if $S \nsubseteq A$ and $T \subseteq B, S \wedge^{A \wedge B} T=S \wedge^{B} T$.

Theorem 2.4. Let $C$ be a coalgebra and $Q_{C}$ its valued Gabriel quiver. Let $A$ and $B$ two subcoalgebras of $C$ whose valued Gabriel quiver are $Q_{A}$ and $Q_{B}$, respectively. Then the valued Gabriel quiver of $A \wedge^{C} B$ is described as follows:

(1) The set of vertices of $Q_{A \wedge B}$ is the union of the set of vertices of $Q_{A}$ and $Q_{B}$, both viewed as valued subquivers of $Q_{C}$.

(2) Given two simple $A \wedge^{C} B$-comodules $S$ and $T$.

i) If $S \nsubseteq A$ and $T \nsubseteq B$, there is no an arrow in $Q_{A \wedge{ }^{C} B}$ from $T$ to $S$.

ii) If $S \subseteq A$ and $T \subseteq B$, there is an arrow $T \stackrel{(c, d)}{\longrightarrow} S$ in $Q_{A^{\wedge}{ }^{C} B}$ if and only if it is so in $Q_{C}$.

iii) If $S \subseteq A$ and $T \nsubseteq B$, there is an arrow $T \stackrel{(c, d)}{\longrightarrow} S$ in $Q_{A \wedge^{C} B}$ if and only if it is so in $Q_{A}$.

iv) If $S \nsubseteq A$ and $T \subseteq B$, there is an arrow $T \stackrel{(c, d)}{\longrightarrow} S$ in $Q_{A \wedge{ }^{C} B}$ if and only if it is so in $Q_{B}$.

Proof. It follows from Propositions 2.1 and 2.3 .

From the former result we may deduce the following corollaries.

Corollary 2.5. Let $C$ be a coalgebra, $Q_{C}$ its valued Gabriel quiver and $A$ a subcoalgebra of $C$. Then $Q_{A \wedge A}$ is the full valued subquiver of $Q_{C}$ whose set of vertices are the simple $C$-comodules contained in $A$.

Corollary 2.6. Let $C$ be a coalgebra and $A$ a subcoalgebra of $C$. If $C=A \wedge^{C} A$, then $Q_{C}=Q_{A}$ as valued quivers.

Corollary 2.7. Let $C$ be a hereditary coalgebra with separable coradical and $A$ a subcoalgebra of $C$. Then the following conditions are equivalent:

a) $A$ is coidempotent, that is, $A \wedge^{C} A=A$.

b) $A$ is hereditary and $Q_{A}$ is the full subquiver of $Q_{C}$ whose vertices are the simple $A$ comodules.

Proof. Let us assume that $A$ is coidempotent. Then $Q_{A}=Q_{A \wedge A}$ and hence, by Corollary 2.5. $Q_{A}$ is the full quiver of $Q_{C}$ whose vertices are the simple $A$-comodules. Since $C$ is hereditary, by [15, Corollary 2.7], we may consider the inclusions

$$
A_{0} \subseteq A \hookrightarrow T_{A_{0}}\left(A_{1} / A_{0}\right) \subseteq T_{C_{0}}\left(C_{1} / C_{0}\right)=C,
$$


where, by $T_{D}(N)$, we denote the cotensor coalgebra of a coalgebra $D$ over a $D$-bicomodule $N$. By [15, Theorem 2.6], $\left(A_{0}\right)^{\infty} \cong T_{A_{0}}\left(\left(A_{0} \wedge^{C} A_{0}\right) / A_{0}\right)$, where $\left(A_{0}\right)^{\infty}=\cup_{n \geq 0}\left(A_{0}\right)^{n}$ with $\left(A_{0}\right)^{0}=A_{0}$ and $\left(A_{0}\right)^{n}=\left(A_{0}\right)^{n-1} \wedge^{C} A_{0}$ for $n>0$. Now, since $A$ is coidempotent, $\left(A_{0}\right)^{n}=A_{n}$ the $n$th piece of the coradical filtration of $A$ for any $n \geq 0$ and then $A \cong$ $\left(A_{0}\right)^{\infty} \cong T_{A_{0}}\left(A_{1} / A_{0}\right)$. Therefore $A$ is hereditary.

Conversely, by Corollary 2.5, $Q_{A}=Q_{A \wedge A}$. Then $A_{0}=\left(A \wedge^{C} A\right)_{0}$ and $A_{1}=\left(A \wedge^{C} A\right)_{1}$. By [15, Corollary 2.7],

$$
A \subseteq\left(A \wedge^{C} A\right) \hookrightarrow T_{A_{0}}\left(A_{1} / A_{0}\right)
$$

Since $A$ is hereditary, $T_{A_{0}}\left(A_{1} / A_{0}\right) \cong A$ and then $A \wedge^{C} A=A$.

\section{SEMipRime COALGEBRAS}

Let us introduce semiprime coalgebras as a coalgebraic analog to semiprime algebras. We will say that $C$ is semiprime if, whether $C=A \wedge A$, for some subcoalgebra $A$ of $C$, it happens that $C=A$. Obviously, if $C$ is prime, then $C$ is semiprime. Also, it is easy to see that $C$ is semiprime if and only if $C^{*}$ is semiprime.

Lemma 3.1. $C$ is semiprime if and only if it satisfies that, in case $C=\wedge^{n} A$ for some $n \in \mathbb{N}$, then $C=A$.

Proof. It is enough to prove the necessity of that property. But note that, if $n$ is even, $C=\left(\wedge^{n / 2} A\right) \wedge\left(\wedge^{n / 2} A\right)$ and, by hypothesis, $C=\wedge^{n / 2} A$. Similarly, if $n$ is odd, $C=\wedge^{n} A=$ $\wedge^{n+1} A$, and then $C=\wedge^{(n+1) / 2} A$. So we may reduce the parameter until $C=A \wedge A$, and then $C=A$.

Corollary 3.2. Let $C$ be a semiprime coalgebra with finite coradical filtration then $C$ is semisimple.

Proof. By hypothesis, $C=\wedge^{n} \operatorname{soc} C$ for some $n \in \mathbb{N}$. Thus $C=\operatorname{soc} C$ and we are done.

Observe that semiprime coalgebras are not necessarily indecomposable, unlike it happens with prime coalgebras. For instance, a direct sum of simple coalgebras is semiprime non-prime. The following proposition states that the class of semiprime coalgebras is closed under direct sums. Hence, from the point of view of the representation theory, semiprime coalgebras seem to be a more appropriate class than merely prime coalgebras.

Proposition 3.3. The direct sum of semiprime coalgebras is semiprime as well.

Proof. Let $C=\oplus_{i \in I} D_{i}$, where $D_{i}$ is semiprime for any $i \in I$. Let $A$ a subcoalgebra of $C$ such that $C=A \wedge^{C} A$. Then $A=\oplus_{i \in I} A_{i}$, where $A_{i}$ is a subcoalgebra of $D_{i}$ for any $i \in I$. Moreover, $A_{i} \neq 0$ and $D_{i}=A_{i} \wedge \wedge^{D_{i}} A_{i}$ for any $i \in I$. Thus $D_{i}=A_{i}$ for any $i \in I$ and hence $A=C$.

3.1. Localization in semiprime coalgebras. The theory of localization has turned out to be a nice tool for developing the representation theory of coalgebras, see for instance [16], 24] or [33] for some notions and results. Therefore one might ask oneself about apply that theory to our problems concerning semiprime coalgebras. Let us first to characterize semiprime coalgebras by means of its local structure following the spirit of [18, Theorem 
4.3]. For any $i \in I_{C}$, we denote by $e_{i}$ the primitive idempotent in $C^{*}$ which corresponds to the simple $C$-comodule $S_{i}$.

Theorem 3.4. Let $C$ be an arbitrary coalgebra. $C$ is semiprime if and only if the coalgebra eCe is semiprime for any idempotent $e \in C^{*}$ which is the sum of two primitive orthogonal idempotents.

Proof. Let us assume that $C=A \wedge^{C} A$ and $C \neq A$. Let $x \in C \backslash A$. Then (the right $C$-comodule generated by $x)\langle x\rangle$ is finite dimensional. Assume that $E_{1} \oplus \cdots \oplus E_{n}$ is its injective envelope, where $E_{i}$ are indecomposable injective right $C$-comodules. Hence, there exists $i \in\{1, \ldots, n\}$ such that $x e_{i} \neq 0$ and $x e_{i} \notin A$. Indeed, if $x=\sum_{i=1}^{n} \lambda_{i} x_{i}$, where $x_{i} \in E_{i}$ and $\lambda_{i}$ are nonzero scalars, then $x_{i}=x e_{i}$ for any $i$ and, since $x \notin A$, there exists certain $i$ such that $x_{i} \notin A$. Applying the left version of the above procedure to such element $x_{i}$ we may find a nonzero element $y \in C$ such that $e_{j} y e_{i}=y$ and $y \notin A$. Let us consider $e=e_{i}+e_{j}$, since $e C e=e A e \wedge e A e$ and also $y \in e C e \backslash e A e, e C e$ is not semiprime. The converse may be proven as in [18, Theorem 4.2].

By a similar reasoning we may mend, partially, a gap in the proof of [18, Theorem 4.3]. There, the authors attend to the localization techniques in the context of prime subcoalgebras of path coalgebras. Nevertheless, in its proof, it is only proven that $C=$ $A \wedge B$ and $x \in C \backslash A$ yields $x \in B$. Then, obviously, the proof is not completed. In the following proposition we prove a version of [18, Theorem 4.3] for arbitrary coalgebras. A coalgebra is said to be socle-finite [33] whether its socle is finite dimensional, equivalently, whether the set of pairwise non-isomorphic simple comodules is finite.

Proposition 3.5. An arbitrary coalgebra is prime if and only any socle-finite "localized" coalgebra is prime.

Proof. Let $C$ be an arbitrary coalgebra such that any "localized" coalgebra $e C e$ is prime. Assume that $C=A \wedge^{C} B$ with $C \neq A$ and $C \neq B$. Then there exist nonzero $x \in C / A$ and $y \in C / B$. Proceeding as in Theorem 3.4, there exist $i, j, k, l \in I_{C}$ such that $e_{i} x e_{j}=x$ and $e_{k} y e_{l}=y$. Let $e=e_{i}+e_{j}+e_{k}+e_{l}$. Then $e C e=e A e \wedge e B e$ with $e C e \neq e A e$ and $e C e \neq e B e$. Hence $e C e$ is not prime, a contradiction.

Then, following the former proof, we should only consider "localized" coalgebras with at most four non-isomorphic simple comodules.

\subsection{Shape of the valued Gabriel quiver.}

Theorem 3.6. The following assertions hold:

i) Let $C$ be a semiprime coalgebra, then each connected component of the right (left) valued Gabriel quiver of $C$ is strongly connected.

ii) Let $C$ be a hereditary coalgebra whose right (left) valued Gabriel quiver is strongly connected, then $C$ is prime.

As a consequence, any hereditary semiprime coalgebra is the direct sum of prime coalgebras

Proof. i) Let $S_{i}$ and $S_{j}$ be two simple right $C$-comodules in the same connected component of $Q_{C}$. Then there exists a minimal (non-oriented) path

$$
S_{1}=S_{i}-S_{2} \longleftarrow-\cdots-S_{n-1}-S_{j}
$$


in $Q_{C}$, where $S_{i} \longrightarrow S_{i+1}$ represents $S_{i} \longrightarrow S_{i+1}$ or $S_{i} \longleftarrow S_{i+1}$. Let us prove that, for any $i=1, \ldots n-1$, there exists a path in $Q_{C}$ from $S_{i}$ to $S_{i+1}$. Indeed, if there is an arrow $S_{i} \longrightarrow S_{i+1}$, we are done. If not, there exists an arrow $S_{i} \longleftarrow S_{i+1}$ and then $S_{i+1}$ is a predecessor of $S_{i}$, i.e., $\operatorname{Rad}_{C}\left(E_{i}, E_{i+1}\right)=\operatorname{Hom}_{C}\left(E_{i}, E_{i+1}\right) \neq 0$.

We consider the idempotent $e \in C^{*}$ associated to the simple comodules $S_{i}$ and $S_{i+1}$, and its "localized" coalgebra $D=e C e . \operatorname{Then}^{\operatorname{Hom}_{D}}\left(\bar{E}_{i}, \bar{E}_{i+1}\right) \cong \operatorname{Hom}_{C}\left(E_{i}, E_{i+1}\right) \neq$ 0 , where $\left\{\bar{E}_{i}\right\}_{i \in I_{D}}$ is a complete set of pairwise non-isomorphic indecomposable injective $D$-comodules. If $\operatorname{Hom}_{D}\left(\bar{E}_{i+1}, \bar{E}_{i}\right)=0$, there is no arrow in $Q_{D}$ from $S_{i}$ to $S_{i+1}$. So $Q_{D}$ is a subquiver of

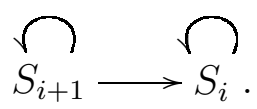

It is not difficult to see that $D=A \wedge A$, where $A=e_{i} D e_{i} \oplus e_{i+1} D e_{i+1}$ (see the proof of [14, Theorem 6.2]), and therefore $D$ is not semiprime and contradicts Theorem 3.4. Hence $0 \neq \operatorname{Hom}_{D}\left(\bar{E}_{i+1}, \bar{E}_{i}\right) \cong \operatorname{Hom}_{C}\left(E_{i+1}, E_{i}\right)$ and $S_{i}$ is a predecessor of $S_{i+1}$. By [24, Theorem 1.9], there exists a path in $Q_{C}$ from $S_{i}$ to $S_{i+1}$.

ii) Let us first suppose that $C$ is colocal. Then $C$ is the unique indecomposable injective right (or left) $C$-comodule. Furthermore, any non-zero morphism of right (or left) $C$-comodules $f: C \rightarrow C$ is surjective, since $C$ is hereditary. Assume that $C=$ $A \wedge^{C} B$, where $A$ and $B$ are proper subcoalgebras of $C$. Hence $0 \neq C / A \cong C^{(\delta)}$ and $0 \neq C / B \cong C^{(\lambda)}$ and therefore there exist a non-zero morphism of right $C$-comodules $f: C \rightarrow C$ such that $f(A)=0$ and a non-zero morphism of left $C$-comodules $g: C \rightarrow C$ such that $g(B)=0$. Now, since $\Delta_{C}(x) \in A \otimes C+C \otimes B$,

$$
(\Delta \circ f)(x)=(f \circ I) \Delta(x) \in C \otimes B
$$

and then, for any $x \in \operatorname{Im} f=C, \Delta(x) \in C \otimes B$. Applying the same reasoning to $g$,

$$
(\Delta \circ g)(x)=(I \circ g) \Delta(x)=0
$$

for any $x \in C$, and we get a contradiction.

Let now $C$ be a hereditary coalgebra where $Q_{C}$ is strongly connected. If $C$ is not prime, $C=A \wedge^{C} B$ with $C \neq A$ and $C \neq B$. We denote by $E_{i}^{C}, E_{i}^{A}$ and $E_{i}^{B}$ the indecomposable injective $C$-comodule, $A$-comodule and $B$-comodule, respectively, with simple socle $S_{i}$. Since $C \neq A$, there exists certain indecomposable injective right $C$-comodule $E_{i}^{C}$ such that $E_{i}^{A} \varsubsetneqq E_{i}^{C}$. Then it may be found a morphism between indecomposable injective right $C$-comodules $f: E_{i}^{C} \rightarrow E_{j}^{C}$ such that $f\left(E_{i}^{A}\right)=0$. Analogously, there exists $g: E_{k}^{C} \rightarrow E_{t}^{C}$ morphism of right $C$-comodules between indecomposable injective $C$-comodules such that $g\left(E_{k}^{B}\right)=0$.

By hypothesis, $Q_{C}$ is strongly connected so there exists a path from $S_{j}$ to $S_{k}$ and a path from $S_{t}$ to $S_{i}$. Then, by [24], $\operatorname{Hom}_{C}\left(E_{t}^{C}, E_{i}^{C}\right) \neq 0$ and $\operatorname{Hom}_{C}\left(E_{j}^{C}, E_{k}^{C}\right) \neq 0$. So let $h: E_{j}^{C} \rightarrow E_{k}^{C}$ and $p: E_{t}^{C} \rightarrow E_{i}^{C}$ be non-zero surjective morphisms of right $C$-comodules. We may consider the surjective morphism $T=p g h f \in \operatorname{End}_{C}\left(E_{i}^{C}\right)$. It verifies that

$$
T\left(E_{i}^{A}\right)=\operatorname{pgh}\left(f\left(E_{i}^{A}\right)\right)=0 \quad \text { and } \quad T\left(E_{i}^{B}\right) \subseteq p g h\left(E_{j}^{B}\right) \subseteq p g\left(E_{k}^{B}\right)=0
$$

Hence, if $e_{i}$ is the primitive idempotent associated to $E_{i}^{C}, 0 \neq e_{i} T \in \operatorname{End}_{e_{i} C e_{i}}\left(e_{i} C e_{i}\right)$ with $e_{i} T\left(e_{i} A e_{i}\right)=e_{i} T\left(e_{i} B e_{i}\right)=0$. Thus $e_{i} A e_{i}$ and $e_{i} B e_{i}$ are proper subcoalgebras 
THE VALUED GABRIEL QUIVER OF A WEDGE PRODUCT AND SEMIPRIME COALGEBRAS 10

of $e_{i} C e_{i}$ and $e_{i} C e_{i}=e_{i} A e_{i} \wedge^{e_{i} C e_{i}} e_{i} B e_{i}$. Therefore, $e_{i} C e_{i}$ is colocal, hereditary and non-prime, a contradiction.

In order to prove the consequence, if $C$ is a hereditary semiprime coalgebra, by $i$ ), each connected component of $Q_{C}$ is strongly connected. By [33], each connected component corresponds to the valued Gabriel quiver of an indecomposable direct sum of $C$. Then these summands are hereditary with strongly connected valued Gabriel quiver. By ii), they are prime.

We recall from [13] that a right $C$-comodule $M$ is said to be strictly quasi-finite if every quotient of $M$ is quasi-finite.

Corollary 3.7 (Weak Eisenbud-Griffith Theorem for coalgebras). Any hereditary semiprime strictly quasifinite coalgebra is serial.

Proof. Let $C$ be a coalgebra as stated above. By the former theorem, $C$ is a direct sum of prime coalgebras. Then each direct summand is prime hereditary and strictly quasi-finite. By [14, Theorem 5.2], $C$ is a direct sum of serial coalgebras. Then $C$ is serial.

Corollary 3.8. Let $C$ be a indecomposable pointed coalgebra. The Gabriel quiver of $C$ is strongly connected if and only if $C$ is an admissible subcoalgebra of a prime path coalgebra.

Proof. By 42, any pointed coalgebra $C$ is isomorphic to an admissible subcoalgebra of a path coalgebra $k Q$, i.e., $C$ contains the set of all the arrows and all the vertices. In particular, $Q_{C}=Q$. Therefore, by Theorem 3.6, $k Q$ is prime if and only $Q=Q_{C}$ is strongly connected.

Remark 3.9. Let us remark that, unlike it happens with hereditary coalgebras, there exist indecomposable semiprime non-prime coalgebras. For instance, let us consider the quiver $Q$ formed by a single vertex $x$ and two different loops $a$ and $b$ starting and ending at $x$. Now, let $A$ be the coalgebra generated by $\left\{x,\left\{a^{n}\right\}_{n>0}\right\}$ and $B$ be the coalgebra generated by $\left\{x,\left\{b^{n}\right\}_{n>0}\right\}$ as vector spaces. Both are isomorphic to the polynomial coalgebra and then prime. Let $R=\left\{x,\left\{a^{n}\right\}_{n>0},\left\{b^{m}\right\}_{m>0}\right\}$. This is a non-prime coalgebra, since $R=A \wedge^{R} B$. Nevertheless, it is semiprime. Indeed, if $R=D \wedge^{R} D$, then $\Delta(d) \in D \otimes R+R \otimes D$. Now, for any $n \in \mathbb{N}, \Delta\left(a^{2 n}\right)=a^{n} \otimes a^{n}+$ other terms. Then $a^{n} \in D$. Analogously, $b^{n} \in D$. Then $D=R$.

Actually, in this way, we may find an infinite family of non-prime semiprime coalgebras. Simply, consider $T_{n}$ the subcoalgebra of $k Q$ generated by all paths of length lower or equal than $n$ and the set $\left\{a^{m}, b^{m}\right\}_{m>n}$.

Observe as well that this example gives a negative answer to the conjecture stated in [25], since $R$ is colocal, cocommutative and infinite dimensional, and, in despite of this, it is not prime.

\section{Applications to Representation theory}

Let us finish the paper dealing with its main aim, i.e., study the representation theory of a semiprime coalgebra. Firstly, we show that the assumption of being a basic coalgebra is not a very restrictive condition since primeness and semiprimeness are preserved under Morita-Takeuchi equivalence. We remind the reader that, following [5] or [29], any coalgebra is Morita-Takeuchi equivalent to a basic one. 
Lemma 4.1. Let $C$ and $D$ be two coalgebras which are Morita-Takeuchi equivalent. Then $C$ is prime (semiprime) if and only if $D$ is prime (semiprime).

Proof. Suppose first that $D$ and $C$ are socle-finite coalgebras. Then, by [21], $C$ and $D$ are strongly equivalent and then $C^{*}$ and $D^{*}$ are Morita equivalent. Thus $C^{*}$ is prime (semiprime) if and only if $D^{*}$ is prime (semiprime) and the statement is proved. For an arbitrary dimension of the socle, let $e$ be an idempotent in $C^{*}$ corresponding to a socle-finite injective $C$-comodule $E$. Let $E^{\prime}$ the injective $D$-comodule image under the Morita-Takeuchi equivalence and $e^{\prime} \in D^{*}$ an idempotent associated to $E^{\prime}$. Then $e C e$ and $e^{\prime} D e^{\prime}$ are socle-finite and Morita-Takeuchi equivalent. By the arguments given above, $e C e$ is prime (semiprime) if and only if $e^{\prime} D e^{\prime}$ is prime (semiprime). Hence, by Theorem 3.4 and Proposition 3.5, $C$ is prime (semiprime) if and only if $D$ is prime (semiprime).

It is clear that simple subcoalgebras of $C$ are prime and, because of this, it is commonly said that prime coalgebras are a generalization of simple coalgebras. Nevertheless, a non-simple prime (resp. semiprime) coalgebra is quite far from being a simple (resp. semisimple) one. For instance, they are infinite dimensional or, moreover, have an infinite coradical filtration. Let us now show that the category of comodules of a semiprime coalgebra lacks certain finiteness conditions. A coalgebra is said to be left (right) semiperfect if all indecomposable injective right (left) $C$-comodules are finite dimensional.

Proposition 4.2. Any semiprime left (right) semiperfect coalgebra is semisimple.

Proof. Assume, contrary to our thesis, that $C$ is not semisimple. Then there exists an non-simple indecomposable injective right $C$-comodule $E_{i}$. Therefore, $E_{i} / S_{i} \neq 0$, and there exists certain simple $C$-comodule $S_{j} \subseteq E_{i} / S_{i}$. We consider the injective right $C$ comodule $E=E_{i} \oplus E_{j}$, and the "localized" coalgebra $D$ given by this injective comodule. Then $D$ is a socle-finite left semiperfect, i.e., it is finite dimensional. Since $D$ is semiprime, $D$ is semisimple. Nevertheless, the quotient functor $T: \mathcal{M}^{C} \rightarrow \mathcal{M}^{D}$ is exact, and then $S_{j} \subseteq T\left(E_{i}\right) / S_{i}$, where $T\left(E_{i}\right)$ is the indecomposable injective $D$-comodule whose socle is $S_{i}$, see [24]. Thus $T\left(E_{i}\right)$ is not simple, and we get a contradiction.

We may go further and prove that any Hom-computable semiprime coalgebra is semisimple. We remind the reader that, following [32], a coalgebra is said to be right Homcomputable if the vector space $\operatorname{Hom}_{C}\left(E, E^{\prime}\right)$ is finite dimensional for any pair of indecomposable injective right $C$-comodules $E$ and $E^{\prime}$. Clearly, any left semiperfect coalgebra is right Hom-computable.

Proposition 4.3. Any semiprime right (left) Hom-computable coalgebra is semisimple.

Proof. Following the proof of Proposition 4.2, it is enough to prove that any "localized" coalgebra relative to an injective $E=E_{i} \oplus E_{j}$, where $E_{i}$ and $E_{j}$ are indecomposable, is semisimple. So let $C$ be semiprime right Hom-computable coalgebra and $E=E_{i} \oplus E_{j}$ be an injective right $C$-comodule. Then $(e C e)^{*} \cong \operatorname{Hom}_{e C e}(e C e) \cong \operatorname{Hom}_{C}(E, E)$ is finite dimensional, where $E=C e$. Then $e C e$ is semisimple.

It's well-known that the class of all finite dimensional algebras over an algebraically field is divided into two disjoint classes. This is called the tame-wild dichotomy, see for example [28], 36] or [37]. On the one side, the class of tame algebras whose finitely 
generated indecomposable modules of a fixed dimension can be recovered by a finite number of one-parameter families. On the other side, the class of wild algebras which verify that a classification of its finitely generated indecomposable modules yields such classification of any finite dimensional algebra. Hence, a complete description is feasible for tame algebras only.

When working on coalgebras, we may define tameness and wildness following the same spirit of above [29] [30]. Nevertheless, the tame-wild dichotomy is still an open problem. These definitions are slightly changed in order to treat the category of finitely cogenerated comodules, see [34] and 35]. This is quite natural since indecomposable injective comodules are commonly infinite dimensional and then they are not considered following the classical notions. We denote by $\mathcal{M}_{f c}^{C}$ the category of finitely copresented right $C$-comodules.

Throughout $k$ will be an algebraically closed field. Then, the coalgebra $C$ is MoritaTakeuchi equivalent to an admissible subcoalgebra of the path coalgebra of its Gabriel quiver, see [42]. Hence we shall assume that $C \subseteq k Q$ for certain quiver $Q$ and $k Q_{1} \subseteq C$. We recall from [34] and [35] that, for any finitely copresented $C$-comodule $N$ with a minimal injective copresentation

$$
0 \longrightarrow N \longrightarrow E_{0} \stackrel{g}{\longrightarrow} E_{1},
$$

the coordinate vector of $N$ is the bipartite vector $\operatorname{cdn}(N)=\left(v_{0} \mid v_{1}\right) \in K_{0}(C) \times K_{0}(C)=$ $\mathbb{Z}^{\left(I_{C}\right)} \times \mathbb{Z}^{\left(I_{C}\right)}$, where $v_{0}=\lg \operatorname{th}\left(\operatorname{soc} E_{0}\right)$ and $v_{1}=\operatorname{lgth}\left(\operatorname{soc} E_{1}\right)$. We also recall that a $C-k[t]_{h}$-bicomodule $L$ is said to be finitely copresented if there is a $C$ - $k[t]_{h}$-bicomodule exact sequence

$$
0 \longrightarrow L \longrightarrow k[t]_{h} \otimes E^{\prime} \stackrel{g}{\longrightarrow} k[t]_{h} \otimes E^{\prime \prime}
$$

such that $E^{\prime}$ and $E^{\prime \prime}$ are socle-finite injective $C$-comodules. Here $h(t)$ is a non-zero polynomial in $k[t]$ and $k[t]_{h}=k\left[t, h(t)^{-1}\right]$ is a rational algebra.

A $k$-coalgebra $C$ is said to be of $f c$-tame comodule type (resp. $k$-tame comodule type) (cf. [29] and [34]) if for every bipartite vector $v=\left(v_{0} \mid v_{1}\right) \in K_{0}(C) \times K_{0}(C)$ (resp. length vector $\left.v \in K_{0}(C)\right)$ there exist $k[t]_{h}-C$-bimodules $L^{(1)}, \ldots, L^{\left(r_{v}\right)}$, which are finitely copresented (resp. finitely generated free $k[t]_{h}$-modules), such that all but finitely many indecomposable right $C$-comodules $N$ in $\mathcal{M}_{f c}^{C}\left(\operatorname{resp} . \mathcal{M}_{f}^{C}\right)$ with $\operatorname{cdn} M=v$ (resp. length $M=v$ ) are of the form $M \cong k_{\lambda}^{1} \otimes_{k[t]} L^{(s)}$, where $s \leq r_{v}, k_{\lambda}^{1}=k[t] /(t-\lambda)$ and $\lambda \in k$.

We recall from [29] that a finite dimensional coalgebra $C$ is $k$-tame if and only if its dual algebra $C^{*}$ is $k$-tame.

A $k$-coalgebra $C$ is of $f c$-wild comodule type (resp. $k$-wild comodule type) if the category $\mathcal{M}_{f c}^{C}\left(\right.$ resp. $\left.\mathcal{M}_{f}^{C}\right)$ is of wild type, that is, if there exists an exact and faithful $k$-linear functor $F: \mathcal{M}_{k Q}^{f} \rightarrow \mathcal{M}_{f c}^{C}$ (resp. $F: \mathcal{M}_{k Q}^{f} \rightarrow \mathcal{M}_{f}^{C}$ ) that respects isomorphism classes and carries indecomposable right $k Q$-modules to indecomposable right $C$-comodules, where $Q$ is the quiver $\circ \equiv \circ$ and $\mathcal{M}_{k Q}^{f}$ is the category of finitely generated right $k Q$-modules.

It is easy to see that if $C$ contains a $k$-wild subcoalgebra, then $C$ is $k$-wild.

Lemma 4.4. [35, Proposition 5.1(b)] Let $C$ be an arbitrary coalgebra. $C$ is fc-tame if and only if any socle-finite "localized" coalgebra of $C$ is fc-tame. 
In general, both definitions of tameness and wildness are unrelated. Nevertheless, as pointed out in [32, when the category $\mathcal{M}_{f}^{C}$ is included in $\mathcal{M}_{f c}^{C}$, or equivalently, all the simple $C$-comodules are finitely copresented, or equivalently, if $E / \operatorname{soc} E$ is quasi-finite for any indecomposable injective $C$-comodule $E$, fc-tameness implies $k$-tameness and $k$ wildness implies fc-wildness. For instance, this holds if $C$ is right strictly quasi-finite [13]. The reader should observe that if a simple $C$-comodule is not finitely copresented, there is an infinite number of arrows ending at a vertex of $Q$. Thus $C$ is $k$-wild, since it contains the finite dimensional coalgebra $k Q_{1}$, where $Q$ is one of the following finite quivers (cf. [29], 30]):

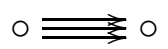

$K_{3}$

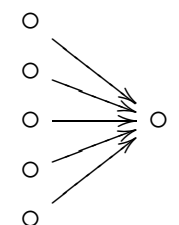

$K_{5}$

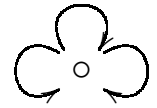

$L_{3}$

Therefore it seems reasonable to assume that $Q$ is locally finite, i.e., any vertex of $Q$ has finite number of arrows starting or ending at it.

Proposition 4.5. Let $C$ be a hereditary semiprime $k$-tame coalgebra. Then $C$ is serial.

Proof. By [19], $Q_{C}$ must be a disjoint union of extended Dynkin quivers. Furthermore, by Theorem 3.6, each connected component of $Q_{C}$ is strongly connected. Then, each connected component of $Q_{C}$ must be $\mathbb{A}_{n}$ for some $n>0$. Then, by [14, Theorem 2.5], $C$ is serial.

Nevertheless, if we drop the hereditariness of the coalgebra, the above statement fails.

Example 4.6. Let $C$ be the subcoalgebra of the path coalgebra $k Q$, where $Q$ is the quiver formed by a single vertex $x$ and two loops $a$ and $b$ starting and ending at $x$, generated by $\left\{x,\left\{a^{n}\right\}_{n>0},\left\{b^{m}\right\}_{m>0}\right\}$. By Remark 3.9, $C$ is semiprime. Also, $C$ is a string coalgebra in the sense of [30], and hence it is of k-tame comodule type. Nevertheless, it is clear that $C$ is not serial, see [14].

The following result will be used in the proof of Theorem 4.8. By a monomial coalgebra we mean a subcoalgebra of a path coalgebra generated by paths as vector space. We also remind from [30] that an admissible subcoalgebra $C$ of a path coalgebra $k Q$ is said to be string if satisfies the following properties:

a) each vertex of $Q$ is the source of at most two arrows and the sink of at most two arrows.

b) $C$ is a monomial coalgebra.

c) given an arrow $\beta: i \rightarrow j$ in $Q$, there is at most one arrow $\alpha: j \rightarrow k$ in $Q$, and at most one arrow $\gamma: l \rightarrow i$ in $Q$ such that $\alpha \beta \in C$ and $\beta \gamma \in C$.

Lemma 4.7. Let $C$ be a monomial admissible subcoalgebra of $k Q$, where $Q$ is the quiver formed by one vertex $x$ and two loops $a$ and $b$. If $C$ is semiprime and $k$-tame, then it is the coalgebra generated by one of the following sets:

a) the paths $\left\{x,\left\{a^{n}\right\}_{n>0},\left\{b^{m}\right\}_{m>0}\right\}$, or

b) the paths $\left\{x,\left\{b(a b)^{t}\right\}_{t \geq 0},\left\{a(b a)^{s}\right\}_{s \geq 0},\left\{(a b)^{n}\right\}_{n>0},\left\{(b a)^{m}\right\}_{m>0}\right\}$.

As a consequence, $C$ is a string coalgebra. 
Proof. Since $C$ is $k$-tame, by the Weak Tame-Wild Dichotomy proved in [29], $C$ is not $k$-wild. Let us suppose that $a^{2} \in C$ and $b a \in C$. Then $C$ contains the subcoalgebra generated by $\left\{x, a, b, a^{2}, b a\right\}$, which is dual to the finite dimensional algebra $k\langle a, b\rangle$ with relations $a^{3}=b^{2}=b a^{2}=a b=0$. By [27], this algebra is $k$-wild and then $C$ is so.

Let us suppose that $a^{2} \in C$ and $b a \notin C$. If $a b \in C$, we may repeat the above arguments and get a contradiction. Now, if the powers of $a$ are bounded, i.e., there exists certain $t \geq 2$ such that $a^{t} \in C$ and $a^{t+1} \notin C$, consider $A=C \cap\left\langle\left\{a^{s} b^{m}\right.\right.$ such that $\left.\left.s<t, m \geq 0\right\}\right\rangle$. Clearly, $A \wedge^{C} A=C$ and $A \neq C$, since $a^{t} \notin A$. Then, $C$ is not semiprime. Similarly, the powers of $b$ are not bounded. Then $C$ is the coalgebra of $a$ ).

Let us suppose that $a^{2} \notin C$ and $b a \in C$. If $a b \notin C$, then $C$ must be generated by paths $b^{m} a$ for $m \geq 0$. Then, $B \wedge^{C} B=C$, where $B=\left\langle\left\{b^{m}\right\}_{m \geq 0}\right\}$, and $C$ is not semiprime. Then $a b \in C$ and, by similar arguments as above, $b^{2} \notin C$. Since $C$ has infinite coradical filtration, i.e., the length of the paths in $C$ is unbounded, $C$ is the coalgebra of $b$ ).

Finally, if $a^{2} \notin C$ and $b a \notin C$, the paths in $C$ has the form $a b^{m}$ for $m \geq 0$ and therefore $C=B \wedge B$, where $B=\left\langle\left\{b^{m}\right\}_{m \geq 0}\right\}$, and it is not semiprime.

It is easy to see that the coalgebras of $a$ ) and $b$ ) are semiprime and string.

Theorem 4.8. Let $Q$ be a locally finite quiver and $C$ a monomial admissible subcoalgebra of $k Q$. If $C$ is a semiprime fc-tame coalgebra, then $C$ is a string coalgebra.

Proof. Since $Q$ is locally finite, the number of arrows ending at any vertex of $Q$ is finite, so each simple $C$-comodule is finitely copresented. Then $\mathcal{M}_{f}^{C}$ is contained in $\mathcal{M}_{f c}^{C}$ and, by the Weak Tame-Wild Dichotomy proved in [29, $C$ is not $k$-wild.

Let us prove that in the quiver $Q$ the vertices are the source of at most two arrows. Suppose, contrary to this, that $x \in Q_{0}$ is the source of three arrows, and we prove that either $C$ is $k$-wild, or a "localized" coalgebra of $C$ is $k$-wild. If the arrows end at the same vertex, $Q$ contains one of the following subquivers $\Gamma$ :

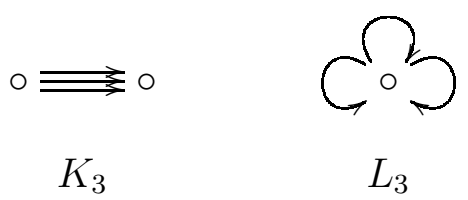

In both cases $(k \Gamma)_{1} \subset C$ is $k$-wild, see [30]. If the arrows end at two different vertices, $Q$ contains one of the following subquivers:

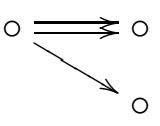

$\widetilde{B}_{2}$

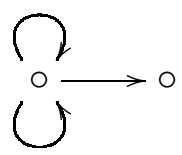

$P_{1}$

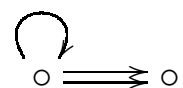

$P_{2}$

$K \widetilde{B}_{2} \subset C$ is a hereditary coalgebra whose Gabriel quiver is not Dynkin diagram and then $k$-wild [29]. For the other two cases, $\left(k P_{i}\right)_{1} \subset C$ is a coradical square complete coalgebra whose separate quiver is not a Dynkin or Euclidean diagram, then, by [20, Theorem 6.5], it is $k$-wild. 
Therefore, we may assume that the three arrows end at three different vertices, i.e, the subquiver has one of the following shapes:

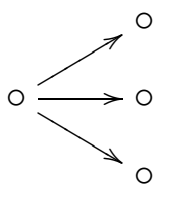

$\Gamma$

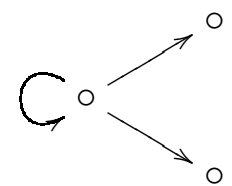

$\Lambda$

Let us consider the "localized" coalgebra $D=e C e$, where $e$ correspond to the vertices of the above subquivers. Then $D$ is also generated by paths, semiprime (Theorem 3.4) and fc-tame [35, Proposition 5.1]. Furthermore, it contains $\Gamma$ or $\Lambda$ as subquiver, see [16]. Now, by Theorem 3.6, the Gabriel quiver of $D$ is strongly connected. Furthermore, if we make use of [20, Theorem 6.5] for analyzing the comodule type of the first piece $D_{1}$ of the coradical filtration, the only possible quivers for $D$ are the following:

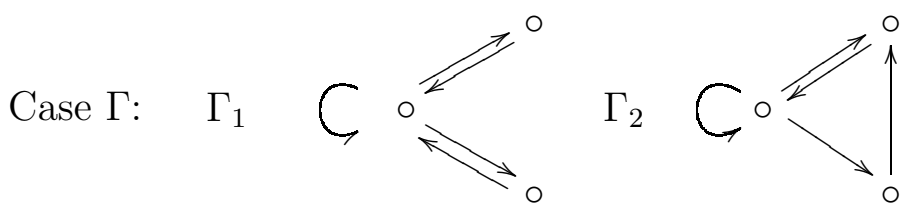

Case $\Lambda$ :
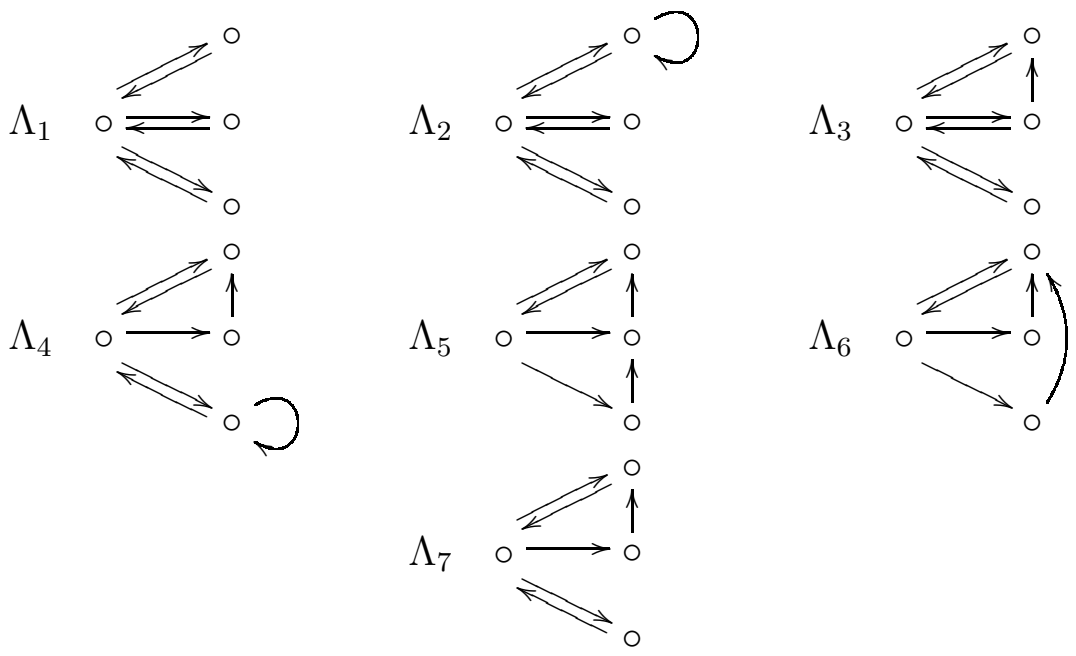

We shall need the following lemma.

Lemma 4.9. If there exists an arrow $\alpha$ such that there is no arrow $\beta$ with $\beta \alpha \in C$ $(\alpha \beta \in C)$, then $C$ is not semiprime. More generally, if there is a path $p$ such that $p \beta \notin C$ $(\beta p \notin C)$ for any arrow $\beta$, then $C$ is not semiprime.

Proof. If there is no arrow $\beta$ such that $\beta \alpha \in C$, then, for each path $p \in C$, or $\alpha \not \leq p$, or $p=\alpha q$ with $\alpha \not \leq q$. We may consider the subcoalgebra

$$
A=C \cap\langle\{\text { paths } p \text { in } C \text { such that } \alpha \not \leq p\}\rangle \text {. }
$$

Since $\alpha \notin A, A \neq C$. Nevertheless, $A \wedge^{C} A=C$. Indeed, for each path $p \in C$, if $\alpha \not \leq p$ then $p \in A$, and, if $p=\alpha q$ with $\alpha \not \leq q$, clearly $\Delta(p) \subseteq A \otimes C+C \otimes A$. Thus $C$ is not semiprime. The proof of the general case is similar. 
Let us consider that the Gabriel quiver of $D$ is $\Gamma_{1}$

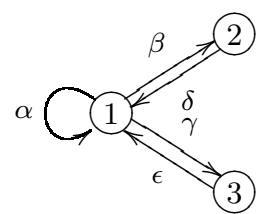

By Lemma 4.9, $\delta \beta \in D$. Then $\delta \beta$ is a cell relative to $\left\{e_{1}, e_{3}\right\}$ in the sense of [16]. Hence, if $e=e_{1}+e_{3}$, by [18], the Gabriel quiver of $e D e$ contains $P_{1}$ as a subquiver, and then $e D e$ is $k$-wild and not $k$-tame, contradicting Lemma 4.4. By a similar reasoning, me may discard all the other possibilities. Then each vertex is the source of at most two arrows. Similarly, it is the sink of at most two arrows.

In the following we will assume that all the quivers involved contain, at most, two arrows starting/ending from/at any vertex. Let us now prove that, if $a$ and $b$ are two arrows starting from certain vertex, and $c$ is an arrow ending at this vertex, then $a c \notin C$ or $b c \notin C$. Actually, following Lemma 4.9, it is proven that $a c \in C$ or $b c \in C$, but not both. In the following list, we describe all possible cases contradicting our thesis and prove that, in these cases, the coalgebra, or a localization of it, must be $k$-wild. By the localization techniques, the reader may assume that the whole quiver is reduced to a quiver whose vertices are those showed in each case.

a) Case $Q=L_{2}$ and $c=a$ or $c=b$. This is proven in Lemma 4.7.

b) Case $\circ \stackrel{c}{\longrightarrow} \circ \underset{b}{\stackrel{a}{\longrightarrow}} \circ$ with $a c, b c \in C$. Then $C$ contains a finite dimensional hereditary coalgebra whose Gabriel quiver is not a Dynkin or Euclidean graph. Then $C$ is $k$-wild.

c) Case $\circ \stackrel{a, b}{\rightleftarrows} \circ$ with $a c, b c \in C$. Then $C$ contains the dual coalgebra of the $k$-wild radical cube zero algebra of [12, Table $\mathrm{W}(2)]$.

d) Case $a$ (1) $\stackrel{b}{\longrightarrow}$ (2) with $a^{2} \in C$ and $b a \in C$. If the powers of $a$ in $C$ are not bounded, then $C$ contains the dual coalgebra of the algebra [12, Table W(6)]. Therefore $a^{t} \notin C$ for some $2<t<7$. Now, since the "localized" coalgebra $e_{1} C e_{1}$ is not simple (and then infinite dimensional) semiprime and fc-tame, its Gabriel quiver should be $L_{2}$ with two loops named $a$ and $\bar{b}$, where $a^{2} \in e_{1} C e_{1}$ but $a^{7} \notin e_{1} C e_{1}$. By Lemma 4.7, this is not possible.

e) Case $a \mathrm{G}_{1} \underset{c}{\stackrel{b}{\rightleftarrows}}(2)$ with $a c, b c \in C$. By Lemma 4.9, $b a c \in C$ or $a^{2} c \in C$. If $a^{2} c \in C$, we are in the dual of Case $d$ ). Therefore $b a c \in C$ and then $b a \in C$. Hence $a^{2} \notin C$, since, otherwise, we are in Case $d$ ). Also, there is no arrow starting at (2). In such a case, since $b a c$ and $a c$ are cells, $e_{2} C e_{2}$ has Gabriel quiver $L_{3}$ or $P_{1}$. Then $C$ contains the subcoalgebra generated by $\{1,2, a, b, c, b a, c b, b c, c b a, c b c\}$ dual to the $k$-wild algebra of [12, Table W(21)].

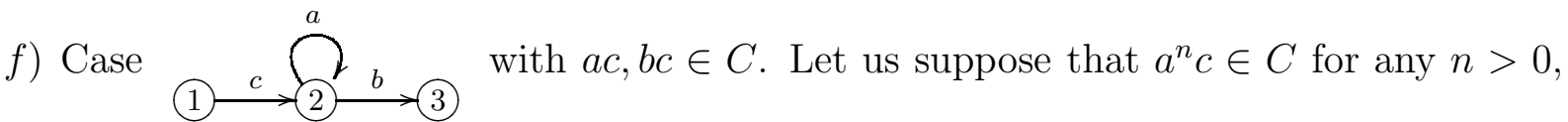
then $C$ is $k$-wild since it contains the hereditary coalgebra of the quiver formed by $c$ and the loop $a$. Hence there exists certain $t>0$ such that $a^{t} c \notin C$ and $a^{t-1} c \in C$. By Lemma 4.9, $b a^{t-1} c \in C$. If $t>1, b a^{2} c \in C$ and $b a^{2} \in C$ so we are in Case $d$ ). Then 
$t=1$, and $b c, b a c \in C$. Now, if there exists another arrow starting at (1), which cannot end at (2), there are three arrows starting at (1) in $e C e$ with $e=e_{1}+e_{3}$. Then, there exists an arrow $d:(3) \rightarrow$ (1). Observe that, if there exists another arrow $h:(3) \rightarrow(1)$, by Lemma 4.9, $c h, c d \in C$ and we may reduce it to Case $b$ ). Furthermore, if there exists a loop at (3), the vertex (3) receives three arrows in $e C e$ with $e=e_{1}+e_{3}$. Therefore, the

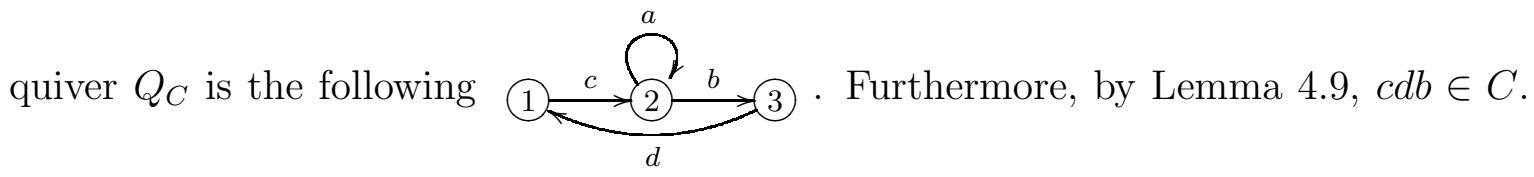
Then, if $e=e_{1}+e_{2}, e C e$ is an admissible coalgebra of obtained from the cell $d b$, and $m c, a c \in C$. Thus, by Case $e$ ), eCe is $k$-wild.

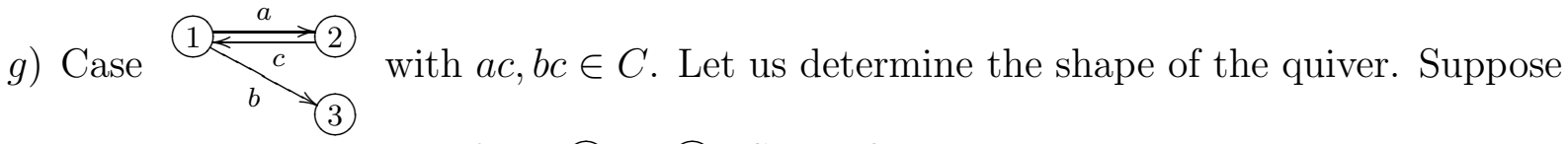
that there is no arrow from (3) to (1). Since $Q$ is strongly connected, there exists an arrow $d$ from (3) to (2). Moreover, any other arrow starting from (3) should be a loop $\alpha$. If such a loop exists, by Lemma 4.9, either $i$ ) there exists a path $d \alpha^{n} b c \in C$ for some $n>0$, or $i i) \alpha^{m} b \in C$ and $d \alpha^{m} \in C$ for any $m>0$. If $i$ ), $e C e$ with $e=e_{1}+e_{2}$ belongs to Case $c$ ), since $d \alpha^{n} b$ is a cell. If $i i$ ), as proved in Case $d$ ), the coalgebra is $k$-wild. If there is no loop $\alpha$, the proof is as in $i$ ). Therefore, $Q$ contains the subquiver (2) $\underset{a}{\stackrel{c}{\rightleftarrows}}(1) \underset{h}{\stackrel{b}{\rightleftarrows}}(3)$. Now, since $a c \in C$, a loop at (2) provokes that $e C e$ is $k$-wild because of its Gabriel quiver contains $P_{1}$, where $e=e_{1}+e_{3}$. Then $Q$ can change from the above quiver uniquely if there is a loop at (3). Let us suppose that there is no such a loop, and the other case may be proven similarly. We have $a h \in C$. Indeed, if $a h \notin C$, there is no path from (3) to (2). Hence, $Q_{e C e}$ is not strongly connected with $e=e_{2}+e_{3}$. Now, by Lemma 4.9, $a h b \in C$ and also $a c \in C$. Then $Q_{e C e}$ is given by ${ }^{\alpha} C_{1} \underset{c}{\stackrel{a}{\rightleftarrows}}$ (2) where $e=e_{1}+e_{2}$ and $\alpha$ corresponds to the cell $h b$. Hence $\alpha c \in e C e$ and $a c \in e C e$. By Case $e$ ), $e C e$ is $k$-wild.

h) Case (1) $\stackrel{c}{\longrightarrow}{ }_{b}^{a}$ (4) $Q$ is strongly connected and (2) is the source of at most two arrows, there exists an arrow $d$ from (3) (or (4)) to (1).

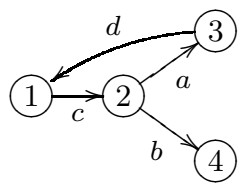

There is no another arrow than $c$ starting at (1). Indeed, if there is a loop in (1), this vertex is the source of three arrows in $e C e$ with $e=e_{1}+e_{3}+e_{4}$, the loop itself and two arrows obtained from the cells $a c$ and $b c$. Similarly, if there is an arrow from (1) to (3) or (4), $Q_{e C e}$ contains the $k$-wild path coalgebra of $\widetilde{B}_{2}$. Finally, if there is an arrow 
$h$ from (1) to (2), by Lemma 4.9, $a h \in C$ or $b h \in C$. Then, it is dual to Case $b$ ). Also, if there is another arrow $h:(3) \rightarrow(1)$, by Lemma 4.9, we are in Case $b$ ).

Let us assume that there is no arrow from (4) to (1). Since $Q$ is strongly connected, there exists an arrow $h:$ (4) $\rightarrow$ (2) or $t:$ (4) $\rightarrow$ (3). If there is no such an $h$, there is no another arrow from (4) to (3) since $Q$ cannot contain $\left(\widetilde{B}_{2}\right)^{o p}$. By Lemma 4.9, if there is a loop $\alpha$ at (4), either $i$ ) $t \alpha^{n} b c \in C$ for some $n \geq 0$, or $\left.i i\right) t \alpha^{m}$ and $\alpha^{m} b \in C$ for any $m>0$. If $i), t \alpha^{n} b$ is a cell relative to the localizing subcategory associated to $e=e_{1}+e_{2}+e_{3}$. Then $e C e$ contains the $k$-wild path coalgebra of the quiver of Case $b$ ). If $i i$ ), as proved in Case $d$ ), $C$ is $k$-wild. If there is not such a loop, the proof is similar to $i i$ ). If there is no such an arrow $t$, we may discuss similarly and, $i$ ) may be reduced to Case $f$ ) and if $i i$ ), the coalgebra is $k$-wild. In case that there exist both, by Lemma 4.9, $t b c \in C$ or $h b c \in C$ and then $t b$ or $h b$ becomes cells for $e=e_{1}+e_{2}+e_{3}$. So $C$ becomes $k$-wild applying the above arguments. Then $Q$ has a (unique) arrow $m:$ (4) $\rightarrow$ (1), and $Q$ contains

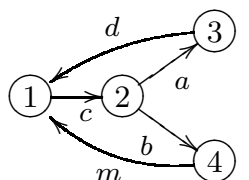

Applying the same arguments, it is not difficult to see that $m b c, d a c \in C$. Then, in the most favorable case, for $e=e_{1}+e_{2}, e C e$ is reduced to Case $c$ ).

Duality, we may prove that if $a$ and $b$ are two arrows ending at certain vertex, and $c$ is an arrow starting from this vertex, then $c a \notin C$ or $c b \notin C$. This completes the proof.

\section{REFERENCES}

[1] E. Abe, Hopf Algebras, Cambridge University Press, 1977.

[2] J. Y. Abuhlail, Fully coprime comodules and fully coprime corings, Appl. Categ. Structures 14 (2006), no. 5-6, 379-409.

[3] J. Y. Abuhlail, A Zariski topology for bicomodules and corings, Appl. Categ. Structures 16 (2008), no. 1-2, 13-28.

[4] Th. Brüstle and Y. Han, Two-point algebras without loops, Comm. Algebra (10) 29 (2001), 46834692.

[5] W. Chin and S. Montgomery, Basic coalgebras, AMS/IP Studies in Advanced Mathematics 1997, 4, $41-47$.

[6] J. Cuadra, Extensions of rational modules, Int. J. Math. Math. Sci. 69 (2003), 4363-4371.

[7] J. Cuadra and J. Gómez-Torrecillas, Idempotents and Morita-Takeuchi theory, Comm. Algebra 30 (2002), 2405-2426.

[8] D. Eisenbud and P. Griffith, Serial rings, J. Algebra 17 (1971), 389-400.

[9] M. Ferrero and V. Rodrigues, On prime and semiprime modules and comodules, J. Algebra Appl. 5(5) 2006, 681-694.

[10] P. Gabriel, Des categories abeliennes, Bull. Soc. Math. France 90 (1962), 323-448.

[11] Y. Han, Controlled wild algebras, Proc. London Math. Soc. 83 (2) (2001), 279-298.

[12] Y. Han, Wild two-point algebras, J. Algebra 247 (2002), 57-77.

[13] J. Gómez-Torrecillas, C. Năstăsescu and B. Torrecillas, Localization in coalgebras. Applications to finiteness conditions, J. Algebra Appl. 6 (2007), 233-243.

[14] J. Gómez-Torrecillas and G. Navarro, Serial coalgebras and its valued Gabriel quivers, J. Algebra 319 (2008), 5039-5059.

[15] P. Jara, D. LLena, L. Merino and D. Ştefan, Hereditary and formally smooth coalgebras, Algebr. Represent. Theory 8 (2005), no. 3, 363-374. 
[16] P. Jara, L. M. Merino and G. Navarro, Localization in tame and wild coalgebras, J. Pure Appl. Algebra 211 (2007), 342-359.

[17] P. Jara, L. Merino, G. Navarro and J. F. Ruíz, Localization in coalgebras, stable localizations and path coalgebras, Comm. Algebra 34 (2006), 2843-2856.

[18] P. Jara, L. Merino, G. Navarro and J. F. Ruíz, Prime path coalgebras, Arab. J. Sci. Eng. 33 (2008), Number 2C, 273-283.

[19] J. Kosakowska and D. Simson, Hereditary coalgebras and representations of species, J. Algebra 293 (2005), 457-505.

[20] J. Kosakowska and D. Simson, Bipartite coalgebras and a reduction functor for coradical square complete coalgebras, Coll. Math. 112 (2008), 89-129.

[21] I-Peng Lin, B. Morita's theorem for coalgebras, Comm. Algebra 1 (1974), no. 4, 311-344.

[22] S. Montgomery, Hopf Algebras and Their Actions on Rings, MBS, No. 82, AMS, 1993.

[23] S. Montgomery, Indecomposable coalgebras, simple comodules, and pointed Hopf algebras, Proc. Amer. Math. Soc. 123 (1995), 2343-2351.

[24] G. Navarro, Some remarks on localization in coalgebras, Comm. Algebra 36 (2008), 3447-3466.

[25] R. Nekooei and L. Torkzadeh, Topology on coalgebras, Bull. Iran. Math. Soc. 27(2) 2001, 45-63.

[26] D. E. Radford, On the structure of pointed coalgebras, J. Algebra 77 (1982), 1-14.

[27] C. M. Ringel, The representation type of local algebras, Proceedings of the International Conference on Representations of Algebras , Carleton Math. Lecture Notes, No. 9, Carleton Univ., Ottawa, Ont., 1974.

[28] D. Simson, Linear Representations of Partially Ordered Sets and Vector Space Categories, Algebra Logic Appl. 4, Gordon \& Breach, 1992.

[29] D. Simson, Coalgebras, comodules, pseudocompact algebras and tame comodule type, Colloq. Math. 90 (2001), 101-150.

[30] D. Simson, Path coalgebras of quivers with relations and a tame-wild dichotomy problem for coalgebras, Lectures Notes in Pure and Applied Mathematics 236 (2005), 465-492.

[31] D. Simson, Irreducible morphisms, the Gabriel-valued quiver and colocalizations for coalgebras, Intern. J. Math. Sci. 72 (2006), 1-16.

[32] D. Simson, Hom-computable coalgebras, a composition factors matrix and the Euler bilinear form of an Euler coalgebra, J. Algebra 315 (2007), 42-75.

[33] D. Simson, Localising embeddings of comodule categories with applications to tame and Euler coalgebras, J. Algebra 312 (2007) 455-494.

[34] D. Simson, Tame-wild dichotomy for coalgebras, J. London Math. Soc. (2) 78 (2008) 783-797.

[35] D. Simson, Tame comodule type, Roiter bocses, and a geometry context for coalgebras, Ukrainian Mathematical Journal 61 (2009), no. 6, 810-833.

[36] D. Simson and A. Skowron'ski, Elements of the Representation Theory of Associative Algebras, Vol. 2: Tubes and concealed algebras of Euclidean type, London Mathematical Society Student Texts 71, Cambridge University Press, Cambridge, 2007.

[37] D. Simson and A. Skowron'ski, Elements of the Representation Theory of Associative Algebras, Vol. 3: Representation-infinite Tilted Algebras, London Mathematical Society Student Texts 72, Cambridge University Press, Cambridge, 2007.

[38] M. E. Sweedler, Hoft Algebras, Benjamin, New york, 1969.

[39] M. Takeuchi, Tangent coalgebras and hyperalgebras. I, Japan. J. Math. 42 (1974), 1-143.

[40] M. Takeuchi, Morita theorems for categories of comodules, J. Fac. Sci. Uni. Tokyo 24 (1977), 629-644.

[41] I. A. Wijayanti and R. Wisbauer, On coprime modules and comodules, Comm. Algebra 37 (2009), no. 4, 1308-1333.

[42] D. Woodcock, Some categorical remarks on the representation theory of coalgebras, Comm. Algebra 25 (1997), 2775-2794.

Department of Computer Sciences and A.I, University of Granada, C. El Greco s/n, E-51002, Ceuta, Spain

E-mail address: gnavarro@ugr.es 